\title{
Immunomodulation by $\alpha(1)$-proteinase inhibitor: lack of chemotactic effects of recombinant human $\alpha(1)$-proteinase inhibitor from yeast on human peripheral blood granulocytes
}

\author{
Birgit Mosheimer, Reinhard Alzner and Christian J. Wiedermann \\ Division of General Internal Medicine, Department of Medicine, Medical University of Innsbruck, A-6020 Innsbruck, Austria
}

Received: 2007.07.24, Accepted: 2007.10.02, Published online first: 2007.12.03

\begin{abstract}
Introduction: Recombinant $\alpha(1)$-proteinase inhibitor, clinically developed for inhalative augmentation therapy in patients with $\alpha(1)$-proteinase inhibitor deficiency or cystic fibrosis, may directly contribute to leukocyte accumulation as it may function as a chemoattractant. The migratory effects of yeast-derived human recombinant $\alpha(1)$-proteinase inhibitor on human peripheral blood neutrophils and eosinophils were therefore tested in vitro.

Materials and Methods: Human peripheral blood leukocytes were prepared from forearm venous blood and tested for migration toward various preparations of yeast-derived recombinant $\alpha(1)$-proteinase inhibitor in modified Boyden-chamber micropore filter assays.

Results: No direct effects of yeast-derived recombinant human $\alpha(1)$-proteinase inhibitor on in vitro migration of isolated neutrophils or eosinophils were seen.

Conclusions: The lack of direct chemotactic effects of recombinant human $\alpha(1)$-proteinase inhibitor despite anti-inflammatory effects in other biological activities of leukocytes may contribute to the preserved antibacterial defense mechanisms observed in patients under experimental augmentation therapy with inhaled $\alpha(1)$-proteinase inhibitor.
\end{abstract}

Key words: neutrophils, eosinophils, chemotaxis, antiproteinase, emphysema.

Corresponding author: Prof. Dr. Christian J. Wiedermann, Department of Medicine, Central Hospital of Bolzano, L. Böhler Street 5, 39100 Bolzano (BZ), Italy, tel.: +39 471 908190, fax: +39 512471 908303, e-mail: Christian.Wiedermann@asbz.it

\section{INTRODUCTION}

In the healthy human lung, $\alpha(1)$-proteinase inhibitor (A1PI) is present at high concentrations and acts as an anti-protease screen to prevent the deleterious effects of free elastase [2]. People with a deficiency of A1PI are at risk for emphysema as a result of low levels of A1PI in the lungs in the presence of neutrophil elastase [25]. In cystic fibrosis airways, A1PI is complexed [3] and proteolytically inactivated [26], resulting in an imbalance of proteases and anti-proteases. The discovery of the structure and function of the A1PI protein and its subsequent isolation and purification have allowed replacement therapy (socalled "augmentation therapy") aimed at preventing the progression of the lung disease associated with deficiency or cystic fibrosis [9]. Isolation of the gene and advances in gene therapy further broadened the potential for specific therapy by the synthesis of recombinant A1PI.
Therapeutic concentrates are prepared from the blood plasma of blood donors and are the only form of A1PI available for therapy so far. All commercially available A1PI products differ from the A1PI directly analyzed in plasma (summarized in Table 1). The observed differences between the products most likely do not play a substantial biologic role because all three products have similar half-lives in humans and neutralize neutrophil elastase to the same extent [12].

To attenuate the deleterious effects of free elastase on pulmonary structure and host defense mechanisms more effectively, the inhalation of A1PI has been proposed as a therapeutic strategy in both A1PI deficiency and cystic fibrosis patients. Inhalation of human plasmatic A1PI increased A1PI levels in adult patients with cystic fibrosis and decreased the levels of elastase activity, neutrophils, pro-inflammatory cytokines, and the numbers of Pseudomonas aeruginosa [7]. Trends of reduced elastase 
Table 1. Human plasmatic $\alpha$-1-antitrypsin preparation modifications ${ }^{1}$

\begin{tabular}{llll}
\hline & \multicolumn{1}{c}{ Aralast $^{\circledR}$} & Prolastin $^{\circledR}$ & Zemaira $^{\circledR}$ \\
\hline Company & Baxter Healthcare & Talecris Biotherapeutics & CSL Behring \\
Plasma half-lives (days) & $5.9 \pm 1.2$ & $5.1 \pm 0.5$ & $5.1 \pm 2.4$ \\
Specific activity (mg functional A1PI/mg protein)* & $\geq 0.55$ & $\geq 0.35$ & $\geq 0.07$ \\
Glycoisoforms & No major difference & & \\
Deamidation (qualitative) & Detectable & 10 & 7 \\
Deamidation (quantitative, $\%)$ & 6 & 2 & 6 \\
C-terminal Lys truncation (\%) & 67 & + & - \\
Cys232 cysteinylation & $+\quad$ Not observed (A1PI fully functional active) & \\
Methionin oxidation & \multicolumn{2}{c}{. } & \\
\hline
\end{tabular}

${ }^{1}$ Data in part taken from product specifications or from Kolarich et al. [12].

* Plasma A1PI, 0.02.

levels and inflammation had already been observed in another study using recombinant A1PI [16].

Recombinant technology has also been used to produce human A1PI [4, 11, 14, 17], as regulatory authorities have recommended the development of recombinant counterparts to blood-derived therapeutic human proteins [18]. Recombinant A1PI is not yet commercially available, but it is under investigation as a therapy for A1PI deficiency for both intravenous application and inhalation. In the development of drug therapy for A1PI deficiency, aerosol administration of recombinant proteins to the airways has been the preferred method of delivery because of their shorter half-lives after intravenous infusion compared with that of A1PI purified and concentrated from human plasma [5].

Recent studies suggest a role for A1PI as an anti-inflammatory modulator [19] independent of its antiproteinase effect, which may explain some of the observations in recent clinical studies. A1PI inhibited lipopolysaccharide (LPS)-induced tumor necrosis factor- $\alpha$ and interleukin (IL)- $1 \beta$ release from monocytes dose dependently and IL-8 release from neutrophils in vitro. Furthermore, A1PI instillation after LPS challenge prevented LPS-induced IL- 8 production in vivo [19]. It is currently unknown to what extend recombinant A1PI affects leukocyte chemotaxis. Alteration of leukocyte migration could have relevance to the cells' pulmonary accumulation, which is of major pathophysiological importance [10]. We therefore investigated the direct effects of human recombinant (rh)A1PI on human peripheral blood granulocyte chemotaxis in vitro. We report that rhA1PI from yeast does not induce in vitro chemotaxis of leukocytes in micropore filter assays.

\section{MATERIALS AND METHODS}

\section{rhA1PI preparation and other material}

The rhA1PI used in this study, supplied by Baxter (Vienna, Austria), was produced by yeast transformed with an expressing plasmid containing a human cDNA encoding the mature normal $\mathrm{Ml}\left(\mathrm{Val}^{213}\right)$ human A1PI protein [21]. This rhA1PI preparation has a molecular mass of about $45 \mathrm{kDa}$, similar to human plasma A1PI, and functions in an identical manner as human plasma rhA1PI as an inhibitor of neutrophil elastase [1].

Formyl-Met-Leu-Phe, dextran, and penicillin-streptomycin were from Sigma Chemical Corp. (St. Louis, MO, USA). RANTES was from Pepro Tech EC Ltd. (London, UK). RPMI 1640 with phenol red purchased from Biochrom AG (Berlin, Germany). Bovine serum albumin (BSA) was from Dade Behring (Marburg, Germany). Lymphoprep $^{\circledR}$ was from Nycomed Pharma AS (Oslo, Norway). MACS separation columns and microbeads were from Miltenyi Biotech (Auburn, CA, USA). Fetal calf serum and phosphate-buffered saline (PBS) were from PAA Laboratories GmbH (Pasching, Austria). Trypsin-EDTA in HBSS from Gibco (Paisley, UK). The microchemotaxis chambers were from Neuroprobe (Bethesda, MD, USA) and the cellulose nitrate filters were from Sartorius (Goettingen, Germany).

\section{Preparation of granulocytes}

Neutrophils and eosinophils were obtained from peripheral EDTA-anticoagulated blood of healthy volunteers by Lymphoprep ${ }^{\circledR}$ density gradient centrifugation followed by dextran sedimentation and hypotonic lysis of contaminating erythrocytes as described previously [6]. The neutrophil preparations yielded above 95\% neutrophils (by morphology in GIEMSA stains) of more than $99 \%$ viability (by trypan dye exclusion). For the eosinophil preparation, the cells were resuspended in 50 $\mathrm{ml}$ of ice-cold MACS buffer (PBS with $5 \mathrm{mmol} / \mathrm{l}$ EDTA and $0.5 \%$ BSA) per $5 \times 10^{7}$ cells and an equal volume of MACS colloidal superparamagnetic microbeads conjugated with monoclonal anti-human CD16 monoclonal antibody was added and incubated $\left(30 \mathrm{~min}, 4^{\circ} \mathrm{C}\right)$. Recommended volumes of ice-cold MACS buffer were added to the cell-microbead mixture and the cell suspension was loaded onto the separation column. The eluate containing CD16-negative eosinophils was collected, washed, resuspended in RPMI 1640/0.5\% BSA, and the separation procedure was repeated to increase purity. The purity of the sorted eosinophils was $98 \%$ as deter- 
mined by morphology and FACS analysis. Contaminating cells were $1 \%$ lymphocytes, $1 \%$ neutrophils and basophils, and negligible numbers of monocytes/macrophages.

\section{Migration assay}

The migration assays were performed using a modified 48-well Boyden microchemotaxis chamber (Neuroprobe, Bethesda, MD, USA) in which a $5-\mu \mathrm{m}-$ -pore-size cellulose nitrate filter separated the upper and lower chambers [6]. Neutrophils or eosinophils were resuspended in RPMI 1640/0.5\% BSA $\left(1 \times 10^{6}\right.$ cells $\left./ \mathrm{ml}\right)$. Fifty $\mu$ l of the cell suspension were placed into the upper chamber and allowed to migrate toward $10 \mathrm{nmol} / \mathrm{l}$ of formyl-Met-Leu-Phe or toward $10 \mathrm{ng} / \mathrm{l}$ of RANTES chemokines, which were used as positive-control chemoattractants at optimal concentrations for neutrophils and eosinophils, respectively; various dilutions of rhA1PI probes 1-4; or respiratory cell culture supernatants placed in the lower chamber for $30 \mathrm{~min}$ (neutrophils) or $60 \mathrm{~min}$ (eosinophils) at $37^{\circ} \mathrm{C}$ in a humidified atmosphere $\left(5 \% \mathrm{CO}_{2}\right)$. After the migration period, the nitrocellulose filters were dehydrated, fixed, and stained with hematoxylin. The migration depth of the cells into the filters was quantified by means of microscopy, measuring the distance $(\mu \mathrm{mm})$ from the surface of the filter to the leading front of three cells. Data are expressed as the chemotaxis index, which is the ratio of the distances of directed and random migration of neutrophils or eosinophils into the nitrocellulose filters.

\section{Statistical methods}

Data are expressed as mean \pm standard error of the mean (SEM). Means were compared by the Mann-
-Whitney U-test and Kruskal-Wallis ANOVA. A difference with $\mathrm{p}<0.05$ was considered to be significant. Statistical analyses were performed using the StatView software package (Abacus Concepts, Berkeley, CA, USA).

\section{RESULTS AND DISCUSSION}

To investigate whether granulocyte recruitment to the lung could be a direct effect of rhA1PI, in vitro neutrophil or eosinophil chemotaxis was assessed. The human plasmatic A1PI protein in a range of concentrations from $0.1-3 \mathrm{mg} / \mathrm{ml}$ had no effect on granulocyte chemotaxis (data not shown). Data from different preparations of yeast rhA1PI and a vehicle (MOCK) preparation which contained the preparation's solution but devoid of the protein are shown for neutrophils (Fig. 1) and for eosinophils (Fig. 2). The positive-control attractants $10 \mathrm{nmol} / \mathrm{l}$ of formyl-Met-Leu-Phe and 10 ng/l of RANTES for neutrophils and eosinophils, respectively, significantly stimulated directed migration. Neither of the rhA1PI preparations affected migration in a significant manner at doses ranging from attomolar to micromolar concentrations. The MOCK preparation had no effect on eosinophil migration. It stimulated neutrophil directed migration to some extent, reaching statistical significance at the highest dose tested, corresponding to the rhA1PI vehicle concentration of $>1 \mu \mathrm{mol} / \mathrm{l}$.

Inhaled rhA1PI may directly contribute to leukocyte accumulation in the lung as human plasmatic A1PI may function as a neutrophil chemoattractant when polymerized [15, 20, 22]. However, no direct effect was seen for rhA1PI on neutrophil or eosinophil chemotaxis.
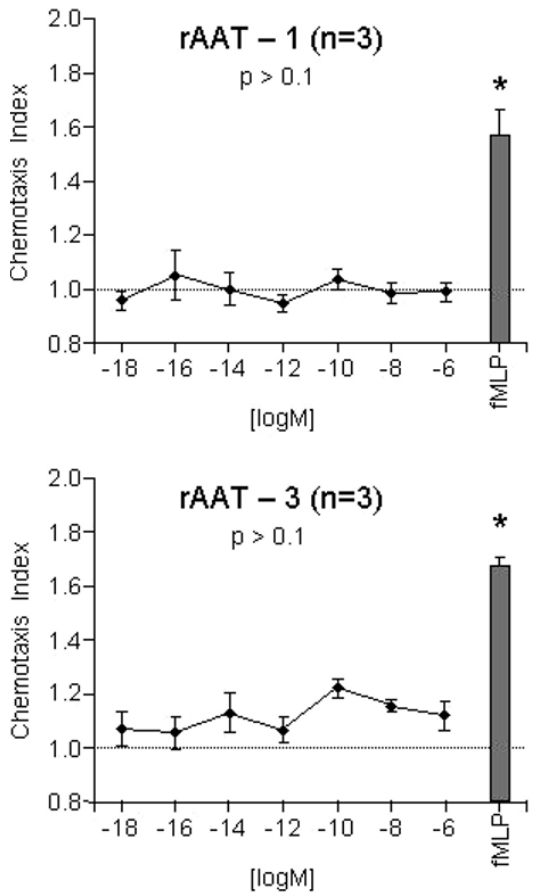

[ $\log \mathrm{M}]$
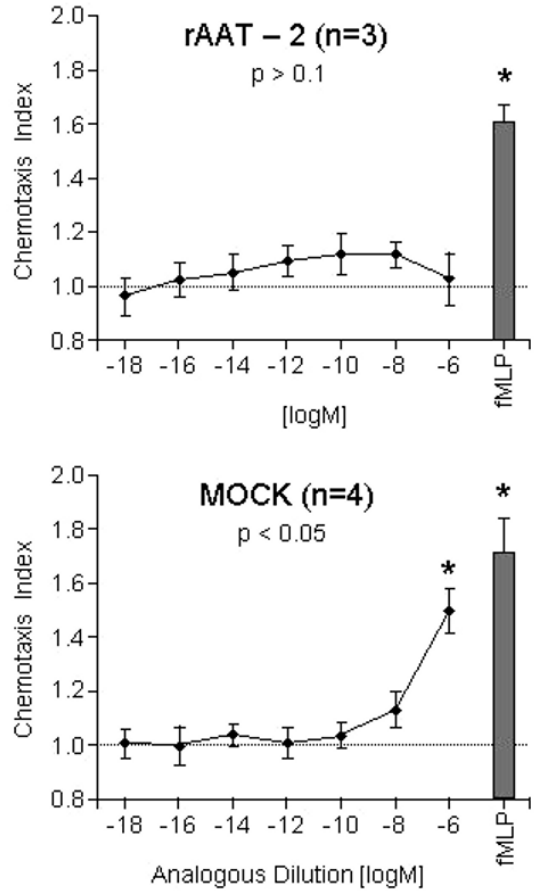

Fig. 1. Human peripheral blood neutrophil migration toward the chemoattractant formyl-Met-Leu-Phe or $\alpha(1)$-antitrypsin (rAAT). Mean distances of random migration were $66,77,74$, and $85 \mu \mathrm{m}$ for rAAT-1, rAAT-2, rAAT-3, and MOC, respectively. $* \mathrm{p}<0.05$, Mann-Whitney U-test versus medium after multiple group comparison by using the Kruskal-Wallis test. 

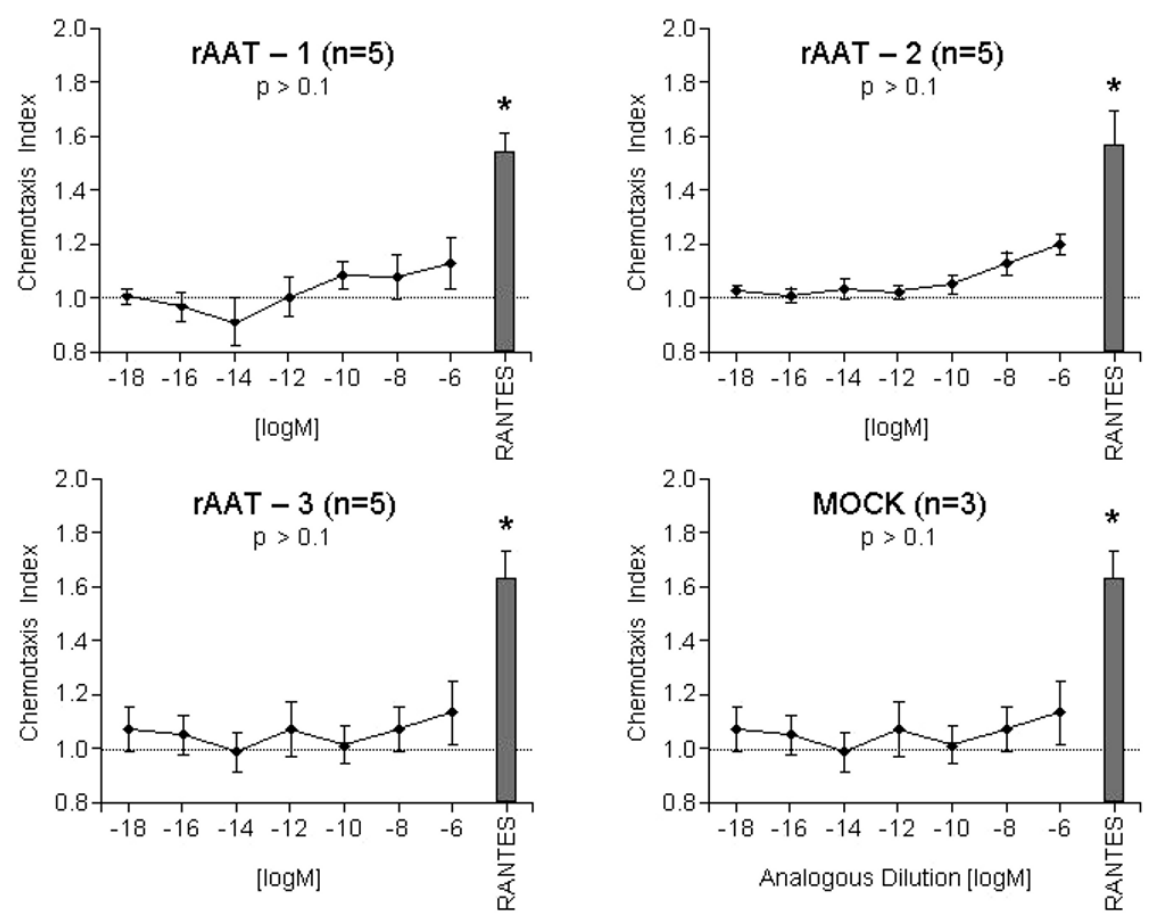

Fig. 2. Human peripheral blood eosinophil migration toward the chemoattractant RANTES or $\alpha(1)$-antitrypsin (rAAT). Mean distances of random migration were 62, 68, 69, and 72 $\mu \mathrm{m}$ for rAAT-1, rAAT-2, rAAT-3, and MOC, respectively. ${ }^{*} \mathrm{p}<0.05$, Mann-Whitney U-test versus medium after multiple group comparison by using the Kruskal-Wallis test.
Polymerization of rhA1PI has not been reported so far; consequently, induction of migration by rhA1PI if polymerized remains speculative. The present results indicate, however, that at high concentrations, a crude preparation of vehicle (MOCK) induced human neutrophil, but not eosinophil migration to some extent, suggesting the potential of eventual contaminants to modify migration. Since eosinophils did not respond to the vehicle, the contaminating compound(s) may be specific and involve molecular mechanisms. As the rhA1PI was unable to induce the same effect, it may be that the dose equivalence between the two types of samples was not given. Another possibility is that the immunomodulatory activity of rhA1PI on leukocytes heterogeneously cross-deactivates chemotactic contamination of the vehicle, such as that described between non-chemotactic tumor necrosis factor-alpha and chemokines [24]. Despite the application of good manufacturing practice, contamination of the yeast-derived rhA1PI formulation may thus potentially induce inflammatory responses after aerosol inhalation. Host responses to yeast are mediated by Toll-like receptor 2 (TLR2), which is normally expressed by a variety of human cells. Cell-surface TLR2 activation is known to induce neutrophil activation. In vivo indirect chemotactic effects may be exerted by induced mediators that are released from respiratory cells, including IL-8, which is a potent granulocyte attractant responsible for leukocyte accumulation in lung airways [8, 13]. Thus for the evaluation of safety, direct and indirect mechanisms by which aerosol inhalation of yeast-derived rhA1PI formulations may adversely affect lung inflammation have to be taken into account.

Given the concerns related to the limited supply of pooled human plasma and the potential for the transmission of infectious agents, some manufacturers are particularly investigating alternative sources of augmentation therapy. Transgenic or recombinant sources could be used in inhalation devices. Yeast expression systems are well suited for the production of rhA1PI. Yeast rhA1PI manufactured in Saccharomyces cerevisiae (baker's or brewer's yeast) [4] was tested in a clinical trial when administered by aerosol inhalation to patients with rhA1PI deficiency associated with hereditary emphysema. According to the sponsors' information, results from this phase $1 \mathrm{~A}$ human study evaluating safety and immunogenicity showed that rhA1PI was well tolerated and a phase $1 \mathrm{~B} / 2 \mathrm{~A}$ clinical trial has been said to be underway to evaluate its effects [1].

rhA1PI from yeast does not induce in vitro chemotaxis of granulocytes in micropore filter assays. Contents in the preparation's vehicle from yeast may still have the potential to induce migration of leukocytes because TLR-2 on other airway cells may indirectly contribute to leukocyte accumulation by release of leukocyte attractants such as IL-8. In consequence, the proinflammatory potential of rhA1PI aerosol therapy will have to be assessed clinically in order to assure its safety. There remains the need for high standards for purification of recombinant proteins.

Strategies to evaluate immune-mediated toxic effects of preparations of transgenic protein should be established to ensure the development of safe and effective therapies. To evaluate the safety of sheep-derived transgenic rhA1PI, aerosolized sheep-derived transgenic rhA1PI was administered in two clinical studies [23]. Systemic antibody responses to non-human protein that was present in very low concentrations in a sheep- 
-derived transgenic rhA1PI formulation were observed after aerosol inhalation. The clinical symptoms and secondary antibody responses suggest that re-exposure could result in intolerance of non-human-protein impurities [23]. These observations may represent an important obstacle to the development of transgenic human proteins for therapeutic use.

In conclusion, the present study explored if rhA1PI directly activates lung inflammatory granulocyte migration. The result of no direct chemotactic effects of A1PI despite its recently described anti-inflammatory affects in other biological activities of leukocytes may explain the preserved antibacterial defense mechanisms observed in patients under experimental inhalative augmentation therapy with rhA1PI.

Acknowledgment: The study was supported in part by the "Verein zur Förderung von Forschung und Fortbildung in klinischer Kardiologie und Intensivmedizin - Innsbruck, Austria”.

\section{REFERENCES}

1. Arriva Pharmaceuticals Inc. http://www.arrivapharm.com/ /clinical-hereditary.php (accessed on Oct 06, 2005).

2. Birrer P. (1995): Proteases and antiproteases in cystic fibrosis: pathogenetic considerations and therapeutic strategies. Respiration, 62 (suppl. 1), 25-28.

3. Birrer P., McElvaney N. G., Rudeberg A., Sommer C. W., Liechti-Gallati S., Kraemer R., Hubbard R. and Crystal R. G. (1994): Protease-antiprotease imbalance in the lungs of children with cystic fibrosis. Am. J. Respir. Crit. Care Med., 150, 207-213.

4. Cabezon T., De Wilde M., Herion P., Loriau R. and Bollen A. (1984): Expression of human alpha 1-antitrypsin cDNA in the yeast Saccharomyces cerevisiae. Proc. Natl. Acad. Sci. USA, 81, 6594-6598.

5. Chughtai B. and O'Riordan T. G. (2004): Potential role of inhibitors of neutrophil elastase in treating diseases of the airway. J. Aerosol Med., 17, 289-298.

6. Feistritzer C., Sturn D. H., Kaneider N. C., Djanani A. and Wiedermann C. J. (2003): Endothelial protein C receptor-dependent inhibition of human eosinophil chemotaxis by protein C. J. Allergy Clin. Immunol., 112, 375-381.

7. Griese M., Latzin P., Kappler M., Weckerle K., Heinzlmaier T., Bernhardt T. and Hartl D. (2007): alpha1-Antitrypsin inhalation reduces airway inflammation in cystic fibrosis patients. Eur. Respir. J., 29, 240-250.

8. Homma T., Kato A., Hashimoto N., Batchelor J., Yoshikawa M., Imai S., Wakiguchi H., Saito H. and Matsumoto K. (2004): Corticosteroid and cytokines synergistically enhance Toll-like receptor 2 expression in respiratory epithelial cells. Am. J. Respir. Cell Mol. Biol., 31, 463-469.

9. Hubbard R. C. and Crystal R. G. (1988): Alpha-1-antitrypsin augmentation therapy for alpha-1-antitrypsin deficiency. Am. J. Med., 84, 52-62.

10. Hubbard R. C., Fells G., Gadek J., Pacholok S., Humes J. and Crystal R. G. (1991): Neutrophil accumulation in the lung in alpha 1-antitrypsin deficiency. Spontaneous release of leukotriene B4 by alveolar macrophages. J. Clin. Invest., 88, 891-897.

11. Jayakumar A., Cataltepe S., Kang Y., Frederick M. J., Mitsudo K., Henderson Y., Crawford S. E., Silverman G.
A. and Clayman G. L. (2004): Production of serpins using baculovirus expression systems. Methods, 32, 177-184.

12. Kolarich D., Turecek P. L., Weber A., Mitterer A., Graninger M., Matthiessen P., Nicolaes G. A., Altmann F. and Schwarz H. P. (2006): Biochemical, molecular characterization, and glycoproteomic analyses of alpha(1)-proteinase inhibitor products used for replacement therapy. Transfusion, 46, 1959-1977.

13. Kurt-Jones E.A., Mandell L., Whitney C., Padgett A., Gosselin K., Newburger P.E., Finberg R. W. (2002): Role of Toll-like receptor 2 (TLR2) in neutrophil activation: GM-CSF enhances TLR2 expression and TLR2-mediated interleukin 8 responses in neutrophils. Blood, 100, $1860-1868$.

14. Ma J. and Cooney C. L. (2004): Application of vortex flow adsorption technology to intein-mediated recovery of recombinant human alpha1-antitrypsin. Biotechnol. Prog., 20, 269-276.

15. Mahadeva R., Atkinson C., Li Z., Stewart S., Janciauskiene S., Kelley D. G., Parmar J., Pitman R., Shapiro S. D. and Lomas D. A. (2005): Polymers of $Z$ alpha1-antitrypsin co-localize with neutrophils in emphysematous alveoli and are chemotactic in vivo. Am. J. Pathol., 166, 377-386.

16. Martin S. L., Downey D., Bilton D., Keogan M. T., Edgar J., Elborn J. S. and Recombinant AAT CF Study Team (2006): Safety and efficacy of recombinant alpha(1)-antitrypsin therapy in cystic fibrosis. Pediatr. Pulmonol., 41, 177-183.

17. McDonald K. A., Hong L. M., Trombly D. M., Xie Q. and Jackman A. P. (2005): Production of human alpha-1-antitrypsin from transgenic rice cell culture in a membrane bioreactor. Biotechnol. Prog., 21, 728-734.

18. Nightingale S. D. (1998): Summary of the Advisory Committee on Blood Safety and Availability Meeting on April 27 and 28, 1998. Washington, D.C.: Department of Health and Human Services. (Accessed April 21, 2005, at http://www.hhs.gov/bloodsafety/summaries/sumapr98.html.)

19. Nita I., Hollander C., Westin U. and Janciauskiene S. M. (2005): Prolastin, a pharmaceutical preparation of purified human alpha1-antitrypsin, blocks endotoxin-mediated cytokine release. Respir. Res., 6, 12.

20. Parmar J. S., Mahadeva R., Reed B. J., Farahi N., Cadwallader K. A., Keogan M. T., Bilton D., Chilvers E. R. and Lomas D. A. (2002): Polymers of alpha(1)-antitrypsin are chemotactic for human neutrophils: a new paradigm for the pathogenesis of emphysema. Am. J. Respir. Cell Mol. Biol., 26, 723-730.

21. Pemberton P. A. and Bird P. (2004): Production of serpins using yeast expression systems. Methods, 32, 185-190.

22. Persson C., Subramaniyam D., Stevens T. and Janciauskiene S. (2006): Do native and polymeric alpha1-antitrypsin activate human neutrophils in vitro? Chest, 129, 1683-1692.

23. Spencer L.T., Humphries J. and Brantly M. L. and Transgenic Human Alpha 1-Antitrypsin Study Group. (2005): Antibody response to aerosolized transgenic human alpha1-antitrypsin. N. Engl. J. Med., 352, 2030-2031.

24. Spilberg I., Mehta J., Muniain M. A., Simchowitz L. and Atkinson J. (1984): Receptor blockade as a mechanism of deactivation of human neutrophils by pepstatin and formyl-Met-Leu-Phe. Inflammation, 8, 73-86.

25. Stoller J. K. and Aboussouan L. S. (2005): Alpha1-antitrypsin deficiency. Lancet, 365, 2225-2236.

26. Suter S. and Chevallier I. (1991): Proteolytic inactivation of alpha 1-proteinase inhibitor in infected bronchial secretions from patients with cystic fibrosis. Eur. Respir. J., 4, 40-49. 